\title{
Influence of the continuum determination method on the mean transmission in the Ly $\alpha$ forest
}

\author{
O. Torbaniuk*
}

Main Astronomical Observatory of the NAS of Ukraine, 27 Akademika Zabolotnoho Str., Kyiv 03680, Ukraine

\begin{abstract}
Determination of the initial flux, or continuum, in the quasar spectra prior to its absorption by the intergalactic $\mathrm{H}_{\mathrm{I}}$ is nontrivial problem and it affects the precision of the mean transmission in the Ly $\alpha$ forest, $\bar{F}(z)$. The results of comparison of the $\bar{F}(z)$ values obtained using different methods of the continuum determination are presented in this paper. This analysis was conducted using the most complete compilation of the $\bar{F}(z)$ data from the literature. It was found that the values of the $\bar{F}(z)$ obtained with the manually determined continuum are systematically higher than those obtained from extrapolated continuum. The difference varies from $5 \%$ at $z=2$ up to $33 \%$ at $z=4.5$, respectively.
\end{abstract}

Key words: quasars: absorption lines, methods: data analysis, statistical, large-scale structure of Universe

\section{INTRODUCTION}

The Ly $\alpha$ forest in the spectra of distant quasars traces the thermal and radiative history of the Universe, as well as the evolution of underlying matter distribution over a wide range of scales and redshifts. It is possible due to relation of the Ly $\alpha$ opacity of the intergalactic neutral hydrogen $\mathrm{H}$ I to its density and other physical parameters. As a measure of opacity the value $F=\mathrm{e}^{-\tau}$ named the transmission is used; here $\tau$ is the optical depth. Fluctuations of this value $\delta_{F}$ provide an invaluable information about the density fluctuations on the smallest scales, available for observations [7, 12, 23, 28, 32, 34].

These studies mainly involve the following steps: (i) determination of the continuum level and normalization the spectrum onto it, (ii) determination of the mean transmission $\bar{F}$, which is a function of the redshift, (iii) calculation of the transmission fluctuations and their two-point statistics (transmission autocorrelation function $\xi_{F}(\Delta v)$ and the flux power spectrum $\left.P_{F}(k)\right)$. The last step is not simple from the math point of view, but it is well understood and unambiguous. The first two steps, involving the data processing, are much more ambiguous and encounter some problems.

One of them is related to the choice of absorptionfree regions within the Ly $\alpha$-forest. Directly it is possible only in the case of high-resolution spectra, when continuum is usually fitted manually and interpolated with spline-polynomials (see e.g. [4, 12, $14,31])$. In the case of median-resolution spectra it is difficult to select unabsorbed regions, therefore indirect techniques based on some assumptions about the quasar spectrum shape are applied. In

*el.torbaniuk@gmail.com

(C) O. Torbaniuk, 2016 the present paper we tried to compile the most complete sample of the $\bar{F}$ values from the literature and analyse the difference between those obtained with different methods of continuum determination.

\section{COMPILATION OF \\ THE $\bar{F}(z)$ MEASUREMENTS}

Our compilation of the $\bar{F}(z)$ measurements from the literature is presented in Figure 1. Short description of these data, including the continuum determination method, is shown in Table 1 . Here we use the term "continuum" for the whole intrinsic spectrum, including emission lines. There are two main classes of methods that are used for this purpose (see, e. g. review in $[20,47])$. One class deals with extrapolation of continuum on wavelengths longer than $1215 \AA$, another class does not use information outside the Ly $\alpha$ forest. Both of these techniques have several modifications and own pros and cons, some of them are noted by the authors of the papers mentioned in Table 1.

For example, in [31] for a sample of eight high resolution quasar spectra with low signal-to-noise ratio $\bar{F}(z)$ was determined in three redshift bins. For this work continuum was obtained manually by common interpolation of the regions free from absorption lines using spline or Chebyshev polynomials. Authors noted some possible sources of continuum errors related to this procedure, e. g. underestimation of continuum at low redshifts $(z \sim 2)$ due to large shallow flux depressions and its overestimation at high redshifts $(z>4)$ because of the high density of absorption lines that prevents accurate reproduc- 
tion of continuum.

Similar problem with high-redshift quasars was also noticed in [22], where authors used own measurements of three quasar spectra with combination of spectra from $[16,24,27,30,33,40]$. The values of $\tau_{\text {eff }}$ were calculated at redshift range $\sim 1.5-4.4$. In addition a compilation of low redshift $(z<1.5)$ data from the HST [1, 17, 18, 35] was used. In [21] the authors obtained the redshift dependence of the $\tau_{\text {eff }}$ at $1.5<z<4$, which is well described by a single power law $\tau_{\text {eff }}(z)=0.0032(1+z)^{3.37 \pm 0.20}$. This gives the value of $\tau_{\text {eff }}(z \approx 0)$ at least four times lower than that from HST observations.

The tendency of systematic underestimation of the zeroth or first-order contribution to the continuum by usual manual fitting methods with multiple splines or other high-order polynomials was also discussed by [38]. They noted, that such continuum underestimates results because of small number of pixels at low flux decrement, therefore they adopted the highest flux value in each individual simulated spectrum as the value of the continuum. Such simple approximation allows to limit from the top the effect of continuum fitting while measuring the flux decrement. This technique was applied by authors for fitting continuum in seven HIRES spectra from [39], for other 14 UVES spectra from this work continuum was fitted manually according to procedure described in [22]. Using combined sample of 21 spectra and after removal of pixels contaminated by metal lines they found the best fit to $\tau_{\text {eff }}(z): \log \tau_{\text {eff }}=$ $\log \tau_{0}+\alpha[(1+z) / 4]$ with $\log \tau_{0}=-0.44 \pm 0.01$, $\alpha=3.57 \pm 0.20$.

In [14] the authors have found that their estimations of the quasar continuum obtained by $\mathrm{Cu}^{-}$ bic spline interpolation are systematically biased low, with the magnitude of the bias increasing from $<1 \%$ at $z=2$ up to $12 \%$ at $z=4$. Authors noted that this bias can be accounted for using mock spectra.

More complicated automatic algorithms in addition to simple interpolation by some polynomials over the unabsorbed regions are applied to highresolution spectra, e.g. the CANDALF software developed by R. Baade and used in [19] for a sample of high-resolution low-redshift quasars. Such software determines continuum simultaneously with the line fitting procedure. Similar idea of adjustment of the continuum level with absorption lines fitting was used in [20].

For low and medium-resolution spectra the continuum fitting procedure is more complicated due to a smaller number of unabsorbed parts that can be seen by eye. In this case some special algorithms are used instead of manual search for unabsorbed parts. One of such techniques was proposed in [9] and applied in [8] using LRIS spectra from [50]. The iterative algorithm consists of multiple fitting of spectrum point by third-order polynomial and rejecting points lying below $2 \sigma$ from the fit.
All the methods of continuum determination within the Ly $\alpha$-forest region that take into account the longer wavelength part of spectrum are based on the idea, that the whole shape of the UV-bump is a result of some general physical processes. The first steps in this direction were made in pioneer work by Press, Rybicki \& Schneider [37]. They obtained $\tau(z)$ using 29 spectra of SSG sample extrapolating continuum shortward of the Ly $\alpha$ emission line in a form $\sim C_{1 / 2} \lambda^{1 / 2}+C_{1} \lambda$. Such extrapolation is useful when the number of unabsorbed pixels in the Ly $\alpha$ forest is too low for high-precision interpolation, that can be caused by either low spectral resolution or low transmission level at high redshifts even in high-resolution spectra.

To avoid this problem for high-redshift quasars the authors of [44] extrapolated continuum in the Ly $\alpha$ region in a sample of 15 spectra at $4<z<6$ with the power law $\sim \lambda^{-1.25}$. Changing the spectral index from -0.75 and -2 they found, that for this range there is a $\pm 18 \%$ range in the spectrum normalization at $1075 \AA$. The same continuum extrapolation, but with spectral index -1.5 , was also applied for four high-redshift quasars in [5]. The data from [44] plotted in Fig. 1 are those averaged over six redshift bins from a combined sample of their own spectra and those from [5]. The same extrapolation was used by [43] for moderate-resolution ESI data with redshift above 4.5 , while in other HIRES and ESI spectra continuum was fitted manually. Comparing the two methods in the redshift range 4.0-4.5 authors have found that the ratio of continuum values for these cases is $0.84 \pm 0.18$, which gives a relatively small correction to the transmission values. Note, that the value of spectral index used in $[5,43,44]$ is lower than that found, e.g. in [49] for a part of quasar composite spectrum redward of $1215 \AA$, but it is more close to values found for a part of the spectrum blueward of the Ly $\alpha$ emission line from the HST and FUSE UV-spectra of the nearest quasars in $[41,46,51]$. On the other hand, for a sample of the 19 most distant quasars with $z$ up to 6.42 the authors of [13] used the spectral slope of $\alpha=1.5$, which is similar to that of [49], which describes the mean quasar continuum in the range of $\sim 1300-5000 \AA$. Extrapolation of the continuum was also used for high-resolution spectra at high redshifts, e. g. for sample of 15 spectra at $4<z<6$ with the power law continuum $\sim \lambda^{-1.25}$ in [44].

More general method based on quasar spectra similarity, and hence involving composite spectra, was proposed for medium-resolution spectra in [6] and used for a sample of 1061 SDSS quasars. In this case the part of a composite spectrum in the Ly $\alpha$ forest region is considered as a multiple of intrinsic quasar spectrum (unabsorbed continuum and emission lines) and the mean transmission $\bar{F}\left(z^{\prime}\right)$ at given redshift $z^{\prime}$. It is clear, that such consideration 
suffers from degeneracy between $\bar{F}\left(z^{\prime}\right)$ and amplitude of continuum, thus the authors had to fix one of them and adopted the unasborbed continuum to be described by a power law extrapolated according to [37]. Later, the same technique was used by [36] for a sample of $2 \mathrm{dF}$ spectra. Similar consideration of spectra as a sum of power-law continuum, extrapolated from longer wavelength region, and several emission lines was used in [11] for a sample of SDSS quasars, but in this case the spectral index was estimated spectrum-by-spectrum. It was noticed, however, that such extrapolation yields the values of $\bar{F}$, which are $\sim 8 \%$ smaller than those obtained from high-resolution spectra (e.g. [11]; see also discussion in $[42,48])$. This discrepancy evidences for overestimation of the continuum level in the case of extrapolation.

More complicated method was proposed in [32]. Instead of using composite spectra the authors of [32] worked with a whole sample of individual spectra. Applying the principal component analysis they found the mean intrinsic spectrum, mean transmission and mean generalized calibration vector, a function defined by them to include calibration errors and mean absorption by metal lines with $\lambda>1300 \AA$, and also their fluctuations.

Recently, [3] introduced a novel technique, which exploits the same idea as in [6], but unlike [6] the authors of [3] used composite spectra to measure the overall shape of the mean flux evolution without fitting continua. To break the degeneracy between $\bar{F}\left(z^{\prime}\right)$ and continuum amplitude the authors derived only the ratio of the mean transmission at different redshifts to its value at $z \sim 2$ and then normalized the results to measurements made from high-resolution data. To account for differences in shape of individual spectra, they adjusted the continuum slope to some reference composite spectrum. However, the authors of [3] point out significant variations of the $\operatorname{Ly} \alpha$ emission line between the composite spectra with different redshift, and relate these variations to redshift errors caused by the line asymmetry due to absorption of its blue side.

Another method of predicting quasar continuum within the Ly $\alpha$ forest region proposed in [45] uses the same idea of correlation of the quasar spectral shape in different parts of spectrum, but do not postulate the constant spectral index over the whole spectrum. Instead of simple extrapolation, the authors applied principal component analysis for 50 low-redshift spectra from the HST and proposed to reconstruct continuum in the range from 1020 to $1216 \AA$ knowing the spectral shape in the range of 1216-1600 $\AA$ using their relation between the weights of principal components for these regions. The reasoning for such method are the correlations between the unabsorbed flux in these two wavelength regions, found by the authors in the HST spectra. The authors tested their method on HST spectra and found an average absolute flux error of $9 \%$, with a range of $3 \%-30 \%$.

\section{THE METHOD OF COMPARISON}

All the data compiled in Fig. 1 and described in the previous section were presented as sets $\left\{z, \Delta_{z}, \tau_{\text {eff }}, \quad \sigma_{\tau}^{\text {up }}, \sigma_{\tau}^{\text {low }}\right\} \quad$ or $\quad\left\{z, \Delta_{z}, \bar{F}, \sigma_{F}^{\text {up }}, \sigma_{F}^{\text {low }}\right\}$, that depend on the results form presented in particular article: in one case authors presented the effective optical depth $\tau_{\text {eff }}$ and in other - mean transmission $\bar{F}$. Here $\Delta_{z}$ is the redshift range in which the value of $F$ was averaged, $\sigma_{\tau}^{\text {up }}, \sigma_{\tau}^{\text {low }}$ and $\sigma_{F}^{\text {up }}, \sigma_{F}^{\text {low }}$ are the upper and lower errors of the corresponding values. For further analysis the all data were recalculated in terms of the $\bar{F}$, and for each value of $\bar{F}$ we found the arithmetic mean error of $\sigma_{F}$ from the upper and lower values.

After this unification two subsamples of the mean transmission values with redshifts range $z>1$ were compiled. In this redshift range the $\bar{F}(z)$ dependence can be described by a power law. The first subsample includes only the results obtained with manual methods of the continuum fitting using absorptionfree regions of spectrum selected "by eye" and interpolated by polynomials (splines, Chebyshev polynomials, etc.). Mainly, the first subsample includes the results from $[10,14,16,21,22,20,24,30,31,33,39$, $40,43,47,50]$. In [10] and [47] the continuum was additionally fixed using the simulated spectra.

The second subsample includes data obtained with extrapolated continuum (as a power law from redward part of spectra with different values of the spectral index [5, 11, 36, 44, 43]).

It should be noted that the data from [14, 39, 47] are presented taking into account absorption of metals that also exist in the intergalactic medium. Determination of the shape of the metal absorption is difficult. Typically, separate search of metals lines in high-resolution spectra, where metal lines are much smaller than the Hydrogen lines, is produced. Unfortunately, in medium or low resolution spectra such procedure is not possible and "statistical" method of the metals impact subtraction is applied. It consists of a determination of the transmission level of the intergalactic medium in the redward part of the spectrum, free from absorption of neutral hydrogen, and further subtracting from the value of transmission in the Ly $\alpha$-forest, which is actually the sum of transmission in the Ly $\alpha$-line and transmission in lines of metals. Since the contribution of the metal absorption is about $1-2 \%$ it can be neglected in present studies of the comparison of methods of continuum fitting. In fact, we started with a calculation without the data from $[14,39,47]$, but since the difference between $\bar{F}(z)$ obtained with and without those data appeared to be about 1-2\%, we decided to those data to our final sample. 
The data from both subsamples were fitted by the dependence $\bar{F}_{\text {theor }}(z)=e^{-\tau_{\text {eff }}(z)}$, where $\tau_{\text {eff }}(z)=$ $\alpha(1+z)^{\beta}, \alpha$ and $\beta$ are free parameters. The values of parameters were determined using the method of maximum likelihood [29], i. e. maximizing of the function $\mathcal{L} \sim \exp \left(-\chi^{2} / 2\right)$, where

$$
\chi^{2}=\sum_{i}\left[\bar{F}_{i}-\bar{F}_{\text {theor }}\left(z_{i}\right)\right]^{2} / \sigma_{F, i}^{2} .
$$

\section{RESULTS AND DISCUSSION}

The best-fit values of $\alpha$ and $\beta$ parameters along with the marginalized $1 \sigma$-errors are given in $\mathrm{Ta}$ ble 2 . In Fig. 2 the best-fit with $1 \sigma$-errors interval and $1,2,3 \sigma$ contours for likelihood function are shown.

From Fig. 2 it can be seen that the values of $\bar{F}$ from the first subsample (manual fitting of the continuum) are systematically higher than that from the second subsample and this difference increases from $5 \%$ at $z=2$ to $33 \%$ at $z=4.5$. Unfortunately, comparison of these methods is impossible on high redshift, since unabsorbed part in the Ly $\alpha$ forest at $z \gtrsim 4$ cannot be selected due to the high density of absorption lines (the so-called "Gunn-Peterson trough" at $z \sim 5-6)$.

There are few main reasons of the such difference between obtained values of the mean transmission. Firstly, the interpolated unabsorbed parts of the spectrum are usually chosen "by eye" without any studies of the form of emission line profiles and other physical parameters. For example, some intensive emission line may be absorbed so its flux is equal to the average flux in the "pure" continuum, that leads to an underestimation of "true" continuum level. Secondly, the extrapolation does not include the emission lines therefore continuum is chosen as the "average" with lines or "pure" continuum extrapolated from the redward region from Ly $\alpha$ line with higher spectral index than in the Ly $\alpha$-forest region.

It is interesting to compare the current result with those from [3]. Its authors use a method which is actually a "mix" of both continuum fitting procedures. Their results are shown in Fig. 2 with diamonds. One can see that values of $\bar{F}(z)$ from [3] are consistent with our fit for the first subsample because the authors of [3] normalized their values onto some mean value $\bar{F}(z)$ at $z=2$ from high-resolution spectra. But on higher redshifts these values are more close to our fit for the second subsample. Since [3] tried to reproduce the full shape of the initial quasar spectra in the Ly $\alpha$-region, this result, probably, indicates that continuum obtained by extrapolation is closer to the real one than that obtained by manual fitting procedure. Certainly, this claim requires independent verification.

\section{ACKNOWLEDGEMENT}

The author is thankful to Dr. Ganna Ivashchenko and Dr. Irina Vavilova for their invaluable help and fruitful discussions. This work has been supported by the Target Programme of Space Research of the NAS of Ukraine for 2013-2016 and by the Swiss National Science Foundation grant SCOPE IZ7370152581.

\section{REFERENCES}

[1] Bahcall J. N., Bergeron J., Boksenberg A. et al. 1993, ApJS, 87, 1

[2] Baldwin J. A. 1993, ApJ, 214, 679

[3] Becker G. D., Hewett P. C., Worseck G. \& Prochaska J. X. 2013, MNRAS, 430, 2067

[4] Becker G. D., Rauch M. \& Sargent W. L. 2007, ApJ, 662, 72

[5] Becker G. D., Fan X., WhiteR.L. et al. 2001, AJ, 122, 2850

[6] Bernardi M., Sheth R. K., SubbaRao M. et al. 2003, AJ, 125, 32

[7] Cappetta M., D’Odorico V., Cristiani S., Saitta F. \& Viel M. 2010, MNRAS, 407, 1290

[8] Croft R.A., Weinberg D.H., BolteM. et al. 2002, ApJ, 581,20

[9] Croft R.A., Weinberg D.H., Pettini M., Hernquist L. \& Katz N. 1999, ApJ, 520, 1

[10] Dall'Aglio A., Wisotzki L. \& Worseck G. 2008, A\&A, 491,465

[11] Desjacques V., Nusser A. \& Sheth R. K. 2007, MNRAS, 374,206

[12] D’Odorico V., Viel M. \& Saitta F. 2006, MNRAS, 372, 1333

[13] Fan X., Strauss M. A., Becker R.H. et al. 2006, AJ, 132, 117

[14] Faucher-Giguère C., Prochaska J. X., Lidz A., Hernquist L. \& Zaldarriaga M. 2008, ApJ, 681, 831

[15] Garzilli A., Bolton J. S., Kim T.-S., Leach S. \& Viel M. 2012, MNRAS, 424, 1723

[16] Hu E. M., Kim T.-S., CowieL. L., Songaila A. \& Rauch M. 1995, AJ, 110, 1526

[17] Impey C. D., Petry C.E. \& Flint K.P. 1999, ApJ, 524, 536

[18] Impey C. D., Petry C. E., Malkan M. A. \& Webb W. 1996, ApJ, 463, 473

[19] Janknecht E., Reimers D., Lopez S. \& Tytler D. 2006, A\&A, 458, 427

[20] Kim T.-S., Bolton J. S., Viel M., Haehnelt M. G. \& Carswell R.F. 2007, MNRAS, 382, 1657

[21] Kim T.-S., Carswell R.F., Cristiani S., D’OdoricoS. \& Giallongo E. 2002, MNRAS, 335, 555

[22] Kim T.-S., Cristiani S. \& D'Odorico S. 2001, A\&A, 373, 757

[23] Kim T.-S., Viel M., Haehnelt M. G., Carswell R.F. \& Cristiani S. 2004, MNRAS, 347, 355

[24] Kirkman D. \& Tytler D. 1997, ApJ, 484, 672

[25] Kirkman D., Tytler D., Lubin D. \& Charlton J. 2007, MNRAS, 376, 1227 
[26] Kirkman D., Tytler D., Suzuki N. et al. 2005, MNRAS, 360,1373

[27] Kulkarni V.P., Huang K., Green R. F. et al. 1996, MNRAS, 279, 197

[28] Lee K. G., Hennawi J. F., Spergel D. N. et al. 2015, ApJ, 799, 196

[29] Lehmann E. L. \& Casella G. 1988, 'Theory of Point Estimation', Springer

[30] Lu L., Sargent W. L., Womble D. S. \& Takada-Hidai M. 1996, ApJ, 472, 509

[31] McDonald P., Miralda-Escudé J., Rauch M. et al. 2000, ApJ, 543, 1

[32] McDonald P., Seljak U., Burles S. et al. 2006, ApJS, 163, 80

[33] Outram P. J., Boyle B. J., Carswell R. F., Hewett P. C. \& Williams R. E. 1999, MNRAS, 305, 685

[34] Palanque-Delabrouille N., Yèche C., Borde A. et al. 2013, A\&A, 559, A85

[35] Penton S. V., Shull J. M. \& Stocke J. T. 2000, ApJ, 544, 150

[36] Polinovskyi G. 2010, in 'WDS 2010, Proc. Contributed Papers. Part III - Physics', eds. J. Šafránková \& J. Pavlû, Matfyzpress, Prague, 163

[37] Press W. H., Rybicki G. B. \& Schneider D. P. 1993, ApJ, 414, 64

[38] Rauch M., Miralda-EscudéJ., Sargent W. L. W. et al.
1997, ApJ, 489, 7

[39] Schaye J., Aguirre A., Kim T.-S. et al. 2003, ApJ, 596, 768

[40] Schaye J., Theuns T., Rauch M., Efstathiou G. \& Sargent W. L. W. 2000, MNRAS, 318, 817

[41] Scott J., Kriss G., Brotherton M. et al. 2003, ASP Conf. Ser., 311, 31

[42] Seljak U., McDonald P. \& Makarov A. 2003, MNRAS, $342, \mathrm{~L} 79$

[43] Songaila A. 2002, AJ, 127, 2598

[44] Songaila A. \& Cowie L. L. 2002, AJ, 123, 2183

[45] Suzuki N., Tytler D., Kirkman D., O’Meara J. M. \& Lubin D. 2004, ApJ, 618, 592

[46] Telfer R. C., Zheng W., Kriss G. A. \& Davidsen A. F. 2002, ApJ, 565, 773

[47] Tytler D., Kirkman D., O’Meara J. M. et al. 2004, ApJ, 617,1

[48] Tytler D., O’Meara J. M., Suzuki N. et al. 2004, AJ, 128, 1058

[49] Vanden Berk D. E., Richards G. T., Bauer A. et al. 2001, ApJ, 122, 549

[50] Viel M., Haehnelt M. G. \& Springel V. 2004, MNRAS, 354,684

[51] Zheng W., Kriss G. A., Telfer R. C., Grimes J. P. \& Davidsen A. F. 1997, ApJ, 475, 469 
Table 1: Description of the data plotted in Fig. 1. The number of spectra in the sample and the point of obtained $\bar{F}(z)$ are designated as $n$ and $p$, respectively. KPE stands for the Echelle spectrograph at Kitt Peak Observatory, MMT is Multiple Mirror Telescope, UCLES/AAT is the University College of London Echelle Spectrograph at the Anglo-Australian Telescope, HIRES is the High Resolution Echelle Spectrometer at the Keck telescope, LRIS is the Low Resolution Imaging Spectrometer at the Keck II telescope, UVES is the UV-Visual Echelle Spectrograph at the VLT telescope, Kast is the double spectrograph on the Shane $3 \mathrm{~m}$ telescope at Lick observatory, HET is Hooby-Eberly Telescope, KP is Kitt Peak 4m MARS spectrograph, MIKE is the Magellan Inamori Kyocera Echelle spectrograph on Magellan; GHRS, FOS and STIS are spectrometers on the HST; 2QZ is the Two-degree Field QSO Redshift Survey. The rows marked with [K01] and [K02] are those, for which the values of $\tau_{\text {eff }}$ were took not from original papers, but

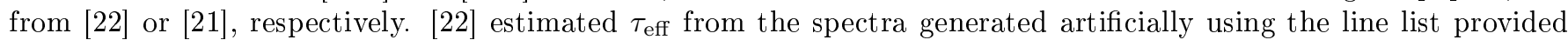
in original papers, except [40]. The continuum approximation technique described in [38], [48] are denoted by [R97], [T04].

\begin{tabular}{|c|c|c|c|c|c|c|}
\hline $\mathrm{n}$ & $\mathrm{p}$ & $R=\lambda / \Delta \lambda$ & origin & $z_{f}$ & continuum & source \\
\hline 29 & - & $\sim 300$ & SSG sample & $2.5-4.3$ & extrap. & Press et al. (1993) [37] \\
\hline 13 & 13 & 180,1300 & FOS & $0-0.95$ & manually & Bahcall et al. (1993) [1] / [K02] \\
\hline 4 & 3 & 36000 & HIRES & $2.74-3.20$ & manually & Hu et al. (1995) [16] / [K01] \\
\hline 1 & 1 & $\sim 45000$ & HIRES & $3.4-4.0$ & manually & Lu et al. (1996) [30] / [K01] \\
\hline 3 & 7 & $\sim 650$ & FOS & $0.52-1.72$ & manually & Impey et al. (1996) [18] / [K02] \\
\hline 1 & 1 & $6000-16000$ & KPE, MMT & $1.67-2.10$ & manually & Kulkarni et al. (1996) [27] / [K01] \\
\hline 1 & 1 & $\sim 38000$ & HIRES & $2.50-2.81$ & manually & Kirkman et al. (1997) [24] / [K01] \\
\hline 10 & 11 & $\sim 1500$ & GHRS & $0.006-0.223$ & manually & Impey et al. (1999) [17] / [K02] \\
\hline 1 & 1 & 35000 & UCLES/AAT & $1.92-2.17$ & manually & Outram et al. (1999) [33] / [K01] \\
\hline 15 & 4 & $\sim 16000$ & GHRS & $0-0.07$ & manually & Penton et al. (2000) [35] / [K02] \\
\hline 8 & 3 & $\sim 45000$ & HIRES & $2.1-4.4$ & manually & McDonald et al. (2000) [31] \\
\hline 9 & 3 & $37500-45000$ & HIRES & $1.85-4.43$ & manually & Schaye et al. (2000) [40] / [K01] \\
\hline 3 & 3 & $\sim 45000$ & UVES & $1.54-2.33$ & manually & Kim et al. (2001) [22] \\
\hline 4 & 4 & $\sim 4700$ & ESI & $5.40-6.16$ & $\alpha=-1.5$ & Becker et al. (2001) [5] \\
\hline 15 & 6 & $\sim 5300$ & ESI & $4.09-5.51$ & $\alpha=-1.25$ & Songaila et al. (2002) [44] \\
\hline 8 & 10 & $\sim 45000$ & UVES & $1.5-3.6$ & manually & Kim et al. (2002) [21] \\
\hline 21 & 42 & $\sim 45000$ & UVES, HIRES & $1.65-4.45$ & manual. / [R97] & Schaye et al. (2003) [39] \\
\hline 1061 & - & $\sim 2000$ & SDSS & $1.65-4.45$ & extrap.+lines (comp.) & Bernardi et al. (2003) [6] \\
\hline 27 & 3 & $\sim 45000$ & UVES (LUQAS) & $2-3$ & manually & Viel et al. (2004) [50] \\
\hline 50 & 60 & 5300,36000 & ESI, HIRES & $2.0-6.3$ & man. $/ \alpha=-1.25$ & Songaila et al. (2004) [43] \\
\hline 77 & 10 & 1200 & Kast/Lick & $1.64-2.36$ & b-spline, corrected & Tytler et al. (2004) [47] \\
\hline 24 & 9 & $\sim 45000$ & HIRES & $2.2-3.2$ & [Т04] & Kirkman et al. (2005) [26] \\
\hline 19 & 98 & 2600 & ESI, MMT, HET, KP & $4.92-6.25$ & $\alpha=-1.5$ & Fan et al. (2006) [13] \\
\hline 9 & 1 & $30000-50000$ & STIS, UVES, HIRES & $0.5-1.9$ & CANDALF & Janknecht et al. (2006) [19] \\
\hline 3492 & 3 & $\sim 2000$ & SDSS & $2.4-3.9$ & extrap.+lines & Desjacques et al. (2007) [11] \\
\hline 74 & 8 & 1300 & FOS & $0-1.6$ & [T04] & Kirkman et al. (2007) [25] \\
\hline 18 & 21 & $\sim 45000$ & UVES (LUQAS) & $1.6-3.6$ & manually & Kim et al. (2007) [20] \\
\hline 86 & 12 & $7000-50000$ & ESI, HIRES, MIKE & $2.0-4.2$ & 3 -spline, corrected & Faucher-Giguere et al. (2008) [14] \\
\hline 40 & 17 & $\sim 45000$ & UVES & $1.7-4.7$ & 3-spline, corr. & Dall'Aglio et al. (2008) [10] \\
\hline 655 & 3 & $500-2000$ & 2QZ & $2.1-2.5$ & extrap.+lines (comp.) & Polinovskiy et al. (2010) [36] \\
\hline 6065 & 28 & $\sim 2000$ & SDSS DR7 & $2.15-4.85$ & - & Becker et al. (2013) [3] \\
\hline 18 & 3 & $\sim 45000$ & UVES & $2.1-2.9$ & - & Garzilli et al. (2012) [15] \\
\hline
\end{tabular}

Table 2: The optimal values of the parameters $\alpha, \beta$ for two subsamples with $1 \sigma$-errors.

\begin{tabular}{c|c|c|c|c}
\hline continuum & $\alpha, \times 10^{-3}$ & $\beta$ & $\chi^{2}$ & d.o.f \\
\hline manually & $3.85 \pm 0.10$ & $3.232 \pm 0.019$ & 68.1 & 121 \\
extrapolation & $4.15 \pm 0.02$ & $3.368 \pm 0.003$ & 70.8 & 27 \\
\hline
\end{tabular}




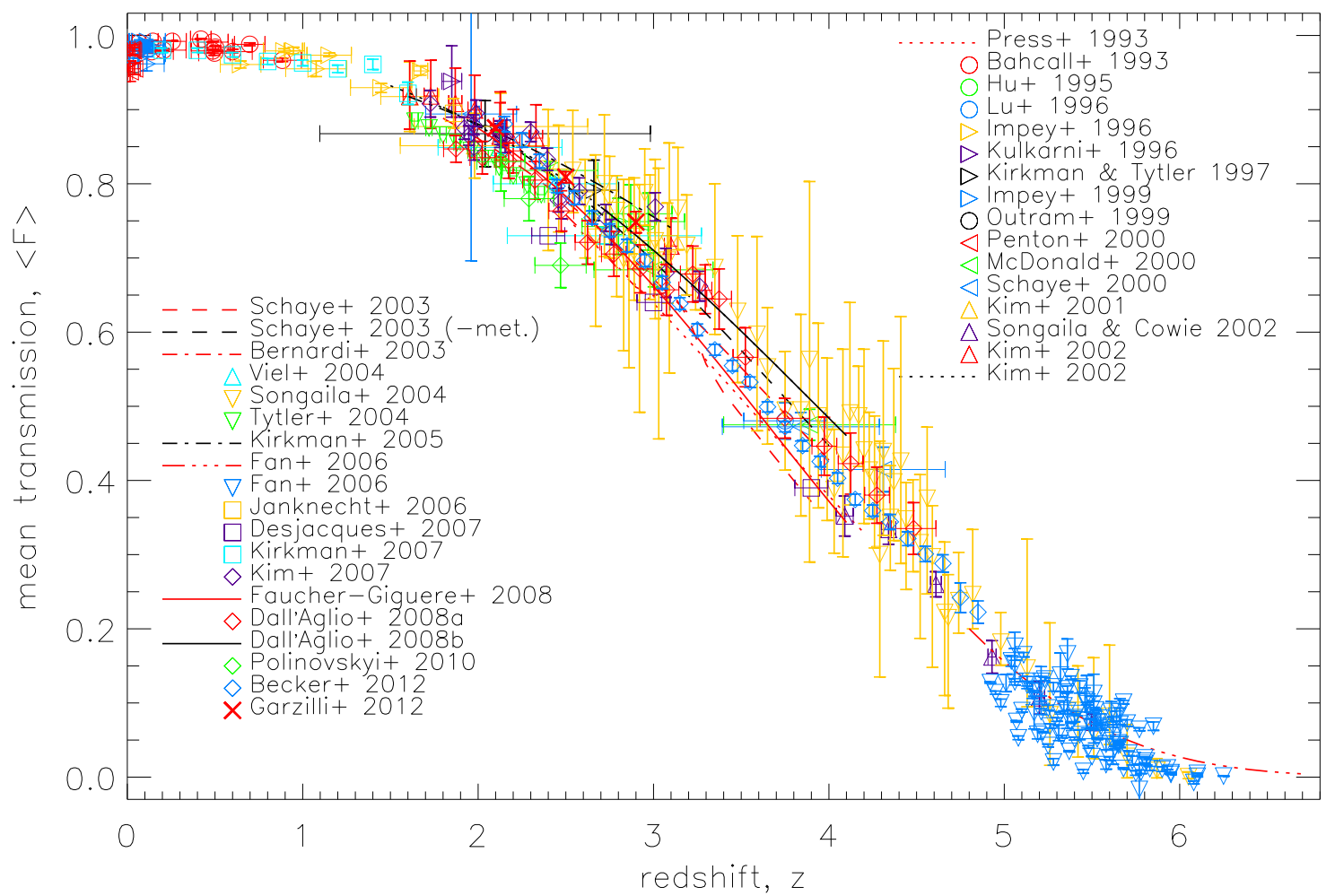

Fig. 1: Compilation of the mean transmission data as a function of redshift from literature. See explanation in Table 1 and in the text.

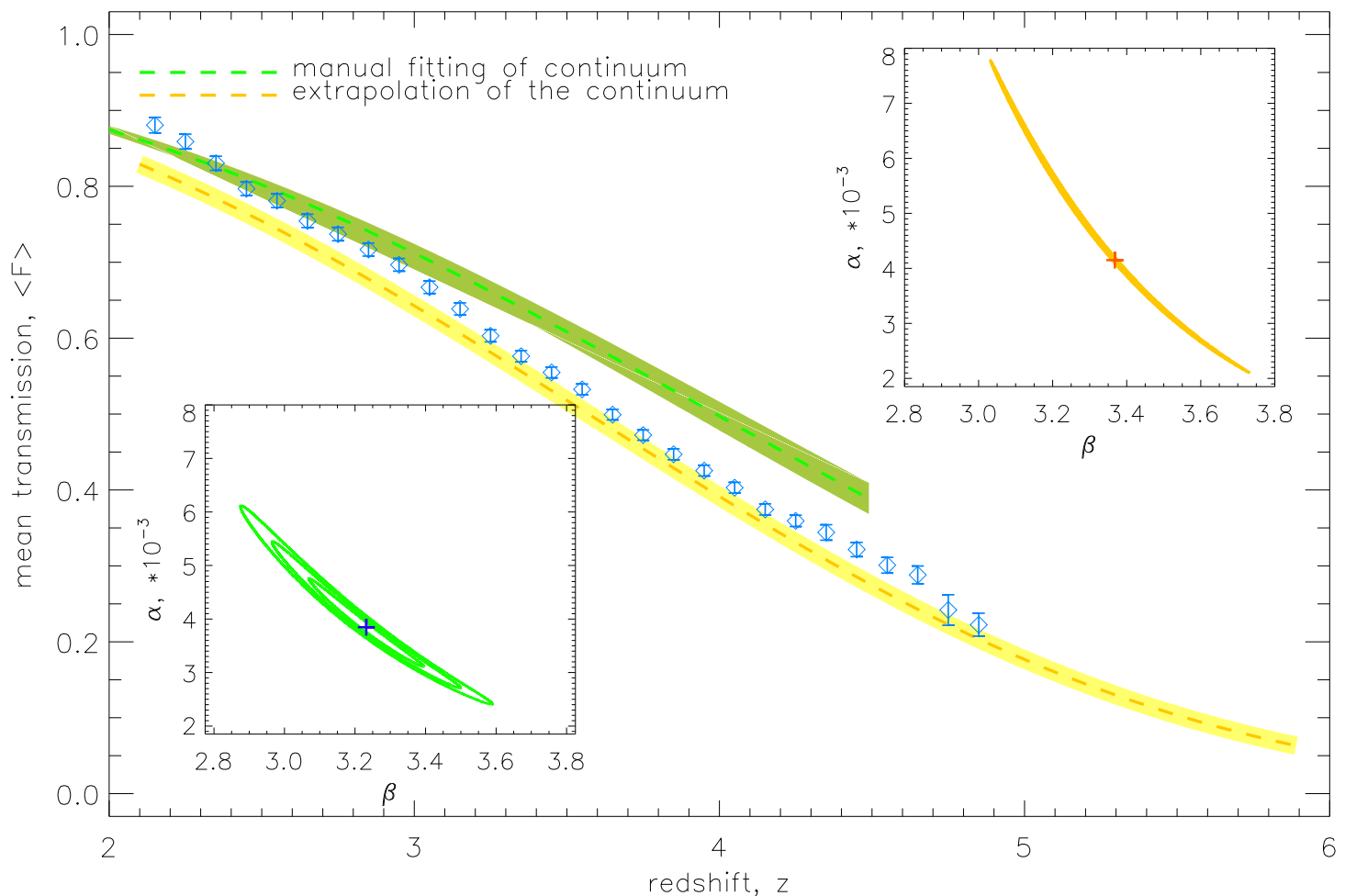

Fig. 2: The optimal approximation with $1 \sigma$-error for the first (green) and second (yellow) subsamples, and the 1,2,3 $\sigma$-level of maximum likelihood function. Diamonds are the results of [3]. 